\title{
Co-transfection of hepatocyte growth factor and truncated TGF- $\beta$ type II receptor inhibit scar formation
}

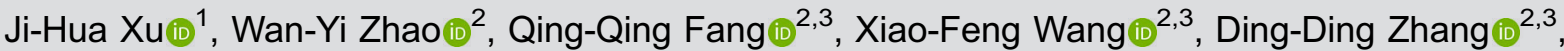 \\ Yan-Yan Hu (i) ${ }^{2,3}$, Bin Zheng (ii) ${ }^{2}$, and Wei-Qiang Tan (iD) ${ }^{1,2}$ \\ ${ }^{1}$ Department of Hand Surgery, The First Affiliated Hospital, Zhejiang University School of Medicine, Hangzhou, Zhejiang, China \\ ${ }^{2}$ Department of Plastic Surgery, Sir Run Run Shaw Hospital, Zhejiang University School of Medicine, Hangzhou, Zhejiang, China \\ ${ }^{3}$ Department of Plastic Surgery, The Fourth Affiliated Hospital, Zhejiang University School of Medicine, Yiwu, Zhejiang, China
}

\begin{abstract}
Wound scarring remains a major challenge for plastic surgeons. Transforming growth factor (TGF)- $\beta$ plays a key role in the process of scar formation. Previous studies have demonstrated that truncated TGF- $\beta$ type II receptor (t-TGF- $\beta$ RII) is unable to continue signal transduction but is still capable of binding to TGF- $\beta$, thereby blocking the TGF- $\beta$ signaling pathway. Hepatocyte growth factor (HGF) is a multifunctional growth factor that promotes tissue regeneration and wound healing. Theoretically, the combination of HGF and t-TGF- $\beta$ RII would be expected to exert a synergistic effect on promoting wound healing and reducing collagen formation. In the present study, lentivirus-mediated transfection of the two genes (t-TGF- $\beta$ RII/ HGF) into fibroblasts in vitro and in a rat model in vivo was used. The results demonstrated that the expression of t-TGF- $\beta$ RII and HGF in NIH-3T3 cells was successfully induced. The expression of both molecules significantly reduced collagen I and III expression, and also inhibited fibroblast proliferation. Furthermore, histological examination and scar quantification revealed less scarring in the experimental wound in a rat model. Moreover, on macroscopic inspection, the experimental wound exhibited less visible scarring compared with the control. Therefore, the present study demonstrated that the combination gene therapy of t-TGF- $\beta$ RII and HGF promoted wound healing, with less scarring and more epithelial tissue formation, not only by suppressing the overgrowth of collagen due to its antifibrotic effect, but also by promoting tissue regeneration.
\end{abstract}

Key words: Wound healing; Truncated transforming growth factor- $\beta$ type II receptor; Hepatocyte growth factor; Scar; Rat; Lentivirus

\section{Introduction}

Scar formation during the process of wound healing remains a challenge for surgeons. Although medical technology has developed rapidly over the past decades, there are few reliable methods to inhibit scar formation. Conventionally, scarring has been considered a natural result of wound healing and is inevitable. As a result, excessive inhibition of scar formation may lead to delayed wound healing. Therefore, an optimal therapeutic approach must focus on suppressing scar formation as well as promoting wound healing.

Previous studies have demonstrated that transforming growth factor (TGF)- $\beta$ plays a key role in the process of scar formation (1), not only by accelerating the proliferation and division of fibroblasts, but also by promoting collagen synthesis. Therefore, reduction of wound TGF- $\beta$ levels or inhibition of the TGF- $\beta$ signaling pathway may prevent scar formation (2,3). Truncated TGF- $\beta$ type II receptor (t-TGF- $\beta$ RII) is a TGF- $\beta$ RII that has lost its serine- and glycine-rich fragments (4). t-TGF- $\beta$ RII is thus unable to continue signal transduction, but is still capable of binding to TGF- $\beta$, thereby blocking the TGF- $\beta$ signaling pathway (5). Hepatocyte growth factor (HGF) is a multifunctional growth factor that promotes mitosis, has anti-apoptotic, anti-fibrotic, and pro-angiogenic properties, whereas it inhibits fibrosis and generation of TGF- $\beta$ in hepatic tissue and enhances collagenase activity (6). In theory, the combination of HGF and t-TGF$\beta$ RII would be expected to exert a synergistic effect on promoting wound healing and reducing collagen formation.

In the present study, both in vitro and in vivo models were used to test this hypothesis. The aim of the study was to determine whether the combination of HGF and t-TGF- $\beta$ RII would be able to reduce the expression of collagen and cell proliferation in vitro and in vivo, hoping to provide a novel approach to gene therapy. 


\section{Material and Methods}

\section{Cell culture}

NIH-3T3 mouse embryonic fibroblasts (Shanghai Institute of Biochemistry and Cell Biology, China) were cultured in Dulbecco's modified Eagle's medium (Gibco; Thermo Fisher Scientific, Inc., USA) supplemented with $10 \%$ fetal bovine serum. The cells were cultured for 4 days at $37^{\circ} \mathrm{C}$ in a humidified incubator with $5 \% \mathrm{CO}_{2}$.

\section{Plasmid construction}

The cDNA codes of human HGF and human TGF- $\beta$ RII were obtained from PubMed (Bethesda, USA). The DNA was amplified and digested by Bam I and Not I enzyme, purified from agarose gel. A truncated human TGF- $\beta$ type II receptor cDNA code was obtained by removing the sequence of signal transduction, as described previously (4). Plasmid vectors (pLV-EFla-EGFP, pLV-EFla-HGFEGFP, and pLV-EFla-tTGF BRII-EGFP) were designed and synthesized by Invitrogen (Thermo Fisher Scientific Inc., China).

\section{Lentivirus package}

293T cells (Thermo Fisher Scientific, Inc., USA) were seeded onto $10-\mathrm{cm}$ plates at a density of $2.0 \times 10^{6} / \mathrm{mL}$ $24 \mathrm{~h}$ prior to transfection. Lentivirus was packaged according to the instructions of HIV package kits when the confluence reached $70-80 \%$. Plasmid pMD2 and psPAX2 were packaged together with target plasmids with a ratio of $2: 1: 2$. A homemade calcium phosphate transfection was used; briefly, the plasmids and calcium phosphate were mixed very well and incubated for $15 \mathrm{~min}$, then added to HEK293T cells. The lentivirus was harvested $24 \mathrm{~h}$ post-transfection and the supernatant was filtered with $0.45 \mu \mathrm{M}$ filters.

\section{NIH-3T3 cell transfection}

NIH-3T3 cells were inoculated into 6-well plates at a density of $2.0 \times 10^{5}$ cells per well. The groups were set as follows: empty virus group (empty lentivirus, $2 \mathrm{~mL} /$ well), t-TGF- $\beta$ RII control group (t-TGF- $\beta$ RII-lentivirus, $2 \mathrm{~mL} /$ well), HGF group (HGF-lentivirus, $2 \mathrm{~mL} /$ well), and experimental group (t-TGF- $\beta$ RII-lentivirus, $1 \mathrm{~mL} /$ well and HGF-lentivirus, $1 \mathrm{~mL} /$ well). The calcium phosphate transfection method was used for transfection, and transfection efficiency and the morphology of $\mathrm{NIH}-3 \mathrm{~T} 3$ was observed 24 and $48 \mathrm{~h}$ after transfection. The transfection efficiency was calculated from percentage of GFP + cells and the average was $75 \%$ (65-85\%).

\section{Cell viability test}

The change in cell proliferation was observed with the Cell Counting Kit-8 (CCK-8) colorimeter (Dojindo Molecular Technologies, Inc., Japan). Briefly, 5000 cells were seeded into a 96-well plate and after 12-h incubation, $50 \mu \mathrm{L}$ of TGF- $\beta 1$ (800 pg/mL) was added to each well and culturing continued for other 48 h. CCK-8 assay followed the protocol.

\section{Western blot analysis}

The protein expression of t-TGF- $\beta$ RII, HGF, collagen I, and collagen III were assayed by western blotting. Total proteins were extracted from each group $96 \mathrm{~h}$ posttransfection. A total of $50 \mu \mathrm{g}$ of protein from each sample was loaded onto a 7.5\% SDS-PAGE gel and electrophoresed at $150 \mathrm{~V}$ for about $1 \mathrm{~h}$. Proteins were then transferred to nitrocellulose membranes $(0.45 \mu \mathrm{m}$, EMD Millipore, USA) that had been blocked with $5 \%$ bovine serum albumin in Tris-buffered saline for $1 \mathrm{~h}$ and washed with TBS-T buffer three times at room temperature. The blots were incubated with primary antibodies against human TGF- $\beta$ RII $(1: 1,000$, Cell Signaling Technology, Inc., USA), HGF (1:1,000, Abcam, USA), collagen type I, or collagen type III $(1: 1,000$, Sigma-Aldrich; Merck KGaA, USA), or GAPDH $(1: 2,000$, Cell Signaling Technology, Inc.) overnight at $4^{\circ} \mathrm{C}$. The membranes were washed three times and then incubated with horseradish peroxidase-conjugated goat anti-rabbit $\lg$ (1:5,000, Pierce; Thermo Fisher Scientific, Inc.) for $1 \mathrm{~h}$ at room temperature. All blots were developed using enhanced chemiluminescence reagents (Supersignal Dura Kit, Pierce; Thermo Fisher Scientific, Inc.) according to the manufacturer's instructions. The signals were captured on X-ray film.

\section{Animal models}

Male Sprague-Dawley rats, 4-6 weeks old and weighing 225-275 g, were used in the present study $(n=24)$. The following experimental procedure was done under anesthesia using $2 \%$ sodium pentobarbital at $35 \mathrm{mg} / \mathrm{kg}$ by intraperitoneal injection. A 3-cm (length) $\times 2-\mathrm{mm}$ (width) rectangular deep incision was made on the dorsal skin, which included the panniculus muscle. A 3-cm (length) $\times$ $3-\mathrm{mm}$ (width) $\times 5-\mathrm{mm}$ (height) trimmed gelatin sponge was inserted into the wound. The rat thick linear scar model was established as described by Wu et al. (7). Forty-eight hours before the animal model was established, $250 \mu \mathrm{L}$ of t-TGF$\beta R$ II-lentivirus and $250 \mu \mathrm{L}$ of HGF-lentivirus were injected subcutaneously under one side of the pre-designed incisions $\left(1 \times 10^{6} \mathrm{pfu} / \mathrm{mL}, \mathrm{n}=6\right)$. A total of $500 \mu \mathrm{L}$ of t-TGF- $\beta$ RII-lentivirus, HGF-lentivirus, empty lentivirus, or DMEM were injected subcutaneously on the other side $\left(1 \times 10^{6} \mathrm{pfu} / \mathrm{mL}, \mathrm{n}=6\right)$. The samples were harvested $14 \mathrm{~h}$ post-transfection. The middle 5-mm portion of the scar was paraffin-embedded, sectioned, stained with hematoxylin and eosin, and observed under a microscope. All animal experiments were approved by the Laboratory Animal Center of Zhejiang University (SYXK 2013-0180).

\section{Histology and qualification of the scar area}

After staining with hematoxylin and eosin, on day 14 tissue sections of the wounds were observed under 
a microscope (Nikon Corporation, Japan) at a fixed magnification $(\times 100)$, and the images were recorded digitally onto a computer with the Image-Pro Plus 6.0 system (Media Cybernetics, USA). The relative area of the wound scar was defined by the 'Irregular AOI' tool and then measured and recorded automatically by the 'Count/Size' tool of Image-Pro Plus (Media Cybernetics, USA).

\section{Statistical analysis}

Data were analyzed using the SPSS 13.0 software (SPSS Inc., USA). The data are reported as means \pm SD. Comparison among the groups was performed using analysis of variance followed by the least significant difference (LSD) and SNK (student-Newman-Keuls) tests. A P-value $<0.05$ was considered to indicate statistically significant differences.

\section{Results}

Higher expression of t-TGF- $\beta$ RII and HGF was observed in vitro and in vivo following transfection

The NIH-3T3 is a well-established cell line for the study of wound healing, and it was used to test our hypothesis in the present study. The expression of t-TGF- $\beta$ RII in the experimental group and control group was found to be notably higher compared with that in other groups, in vitro as well as in vivo (Figure $1 \mathrm{~A}$ and $\mathrm{B}$ ). Moreover, the same result was observed for the expression of HGF, indicating that the transfection was successful, and the model could be used for further study.

t-TGF- $\beta$ RII reduced the expression of collagen I and III

Previous studies have demonstrated that TGF- $\beta$ signaling plays a crucial role in the process of scar formation by accelerating the proliferation of fibroblasts and by promoting collagen synthesis; however, t-TGF- $\beta$ RII inhibits these effects (1). We next tested whether t-TGF$\beta R I$ affects the expression of collagen. In vitro data results revealed that the expression of collagen I was markedly reduced in the experimental group. In addition, the expression of collagen III was reduced in the HGF control, t-TGF- $\beta$ RII, and experimental groups, compared with that in the blank and negative control groups (Figure $1 \mathrm{~A}$ and $\mathrm{B}$ ).

\section{Transfection of t-TGF- $\beta$ RII and HGF inhibited the proliferation of NIH-3T3 cells}

The proliferation of cells transfected with t-TGF- $\beta$ RII/HGF lentivirus was significantly inhibited compared with the control group and single transfected control (Figure 2).

\section{Transfection of t-TGF- $\beta$ RII and HGF inhibited wound scarring and promoted tissue repair}

Our in vitro results indicated that the transfection of t-TGF- $\beta$ RII and HGF inhibited cell proliferation. To further investigate these effects, they were next tested in an established rat model. Both molecules were highly
A

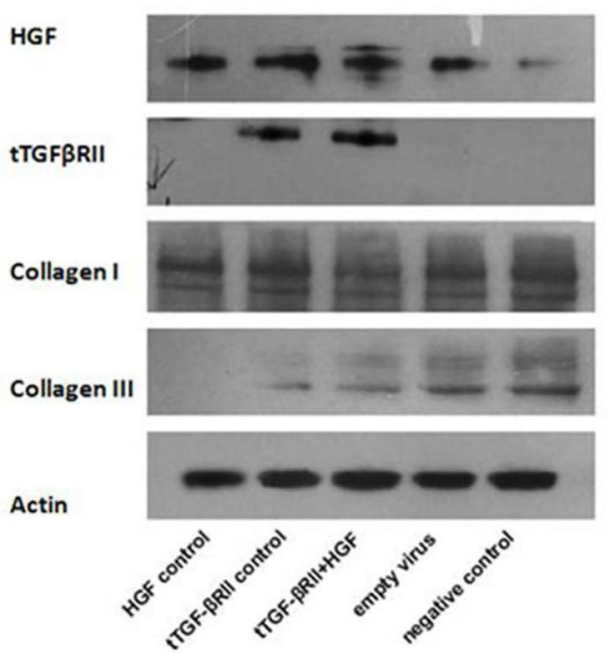

B

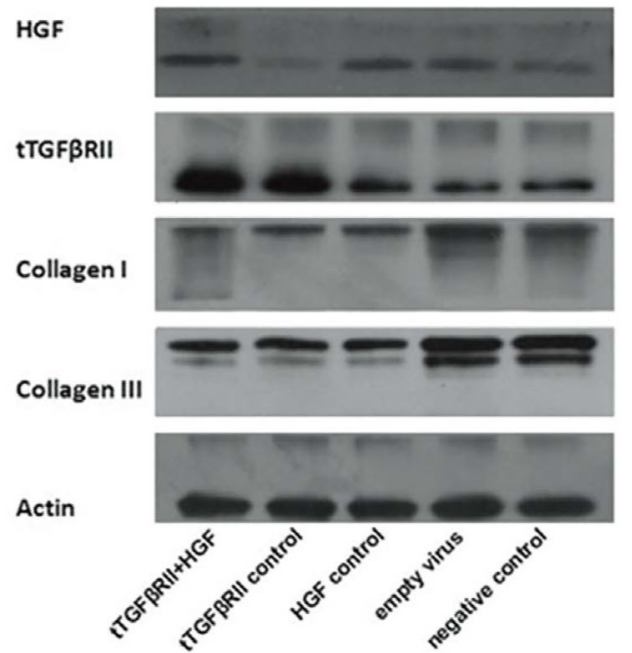

Figure 1. Expression of t-TGF- $\beta$ RII, HGF, and collagen I and III in vitro and in vivo. A, In the in vitro experiment, the expression of collagen III was reduced in HGF control, t-TGF- $\beta$ RII, and experimental groups compared with that in the blank and negative control groups. The result was most prominent in the HGF control group. B, In the in vivo experiment, the expression of collagen I and III was significantly reduced in the HGF control, t-TGF- $\beta$ RII, and experimental groups after 14 days of treatment. t-TGF- $\beta$ RII: truncated transforming growth factor- $\beta$ type II receptor; HGF: hepatocyte growth factor. 


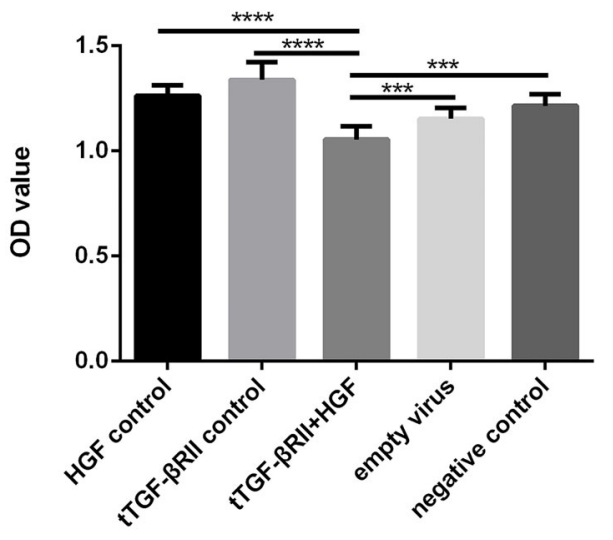

Figure 2. The proliferation of NIH-3T3 cells, which was quantified by Cell Counting Kit- 8 assay, was significantly inhibited by the transfection of t-TGF- $\beta$ RII/HGF-lentivirus, compared with that in the control groups $(P<0.05)$. Data are reported as means $\pm S D$. ${ }^{* * *} \mathrm{P}<0.001$ and ${ }^{* * * *} \mathrm{P}<0.0001$ (least significant difference (LSD) test). t-TGF- $\beta$ RII, truncated transforming growth factor- $\beta$ type II receptor; HGF, hepatocyte growth factor. expressed in the rat model (Figure 1B). Histological examination revealed that the width of the scar was narrower compared with that in the untreated group (Figure 3A-F). Quantification of the wound scar area demonstrated that the wounds in the experimental (t-TGF- $\beta$ RII + HGF), HGF control, and t-TGF- $\beta$ RII groups healed with less scarring compared with those in the empty virus and blank groups. The result was most prominent in the experimental group (Figure 4A). On macroscopic inspection, the treated wounds exhibited less visible scarring compared with the control wounds at 14 days after wounding (Figure 4B).

The above-mentioned results indicated that the combination of t-TGF- $\beta$ RII and HGF mediated by lentivirus can be successfully transfected into fibroblasts. Fibroblast proliferation and fibrosis can be inhibited by the cotransfection of HGF and t-TGF- $\beta$ RII. Therefore, combination gene therapy with t-TGF- $\beta$ RII and HGF promoted wound healing with less scarring and more epithelial tissue formation, not only by suppressing the overgrowth of collagen, due to its antifibrotic effect, but also by promoting tissue regeneration.
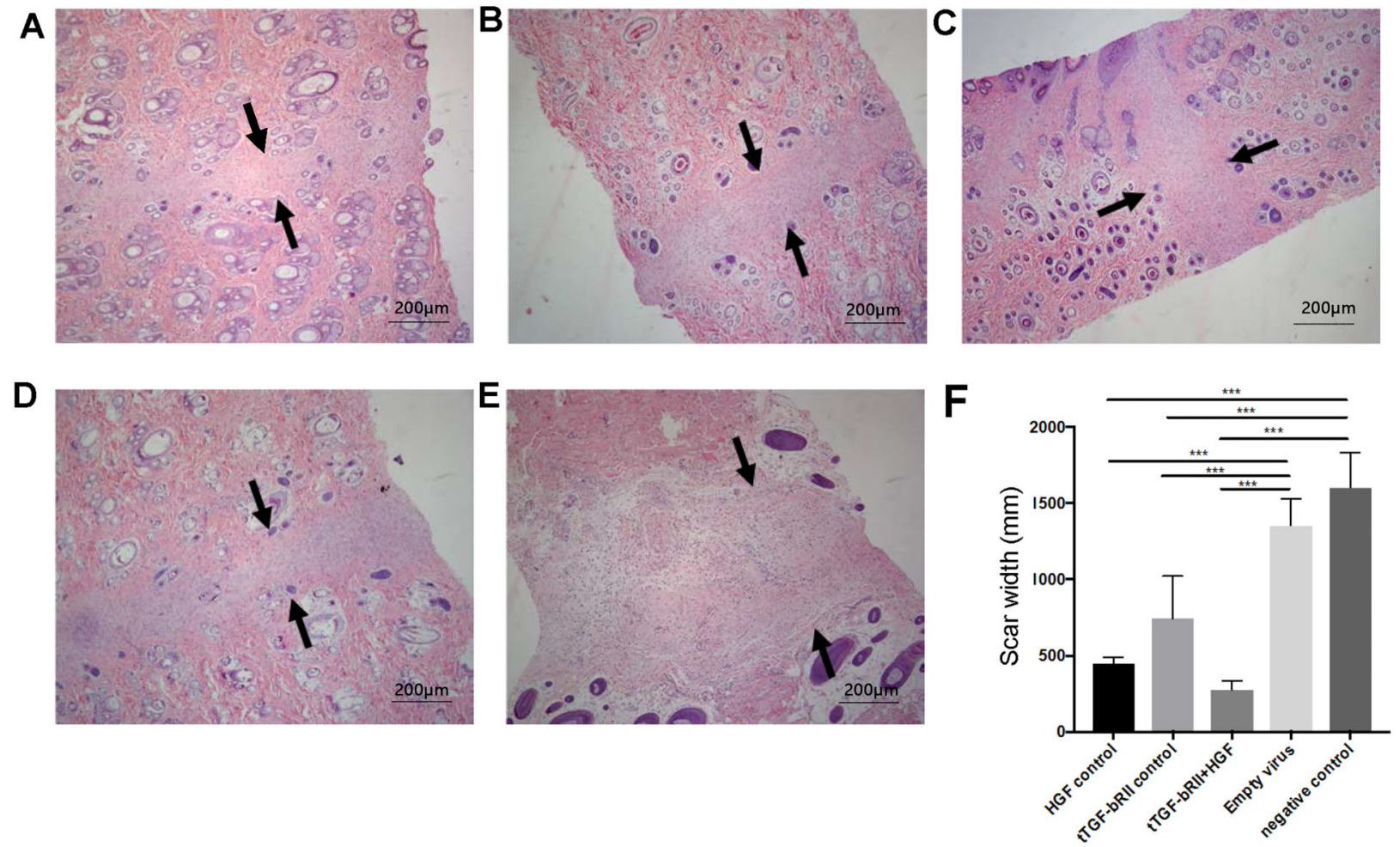

Figure 3. Scar width in each group. Histological examination revealed that the width of the scar was narrower in the experimental wound (hematoxylin and eosin staining; original magnification: $\times 100$, bars: $200 \mu \mathrm{m}$ ). Arrows indicate the scar width. A, Experimental group

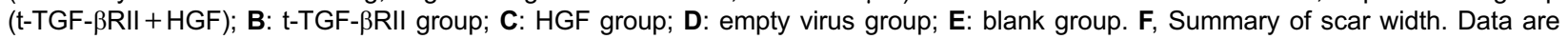
reported as means $\pm \mathrm{SD}$. ${ }^{* * *} \mathrm{P}<0.001$ (Student-Newman-Keuls test). t-TGF- $\beta$ RII: truncated transforming growth factor- $\beta$ type II receptor; HGF: hepatocyte growth factor. 
A

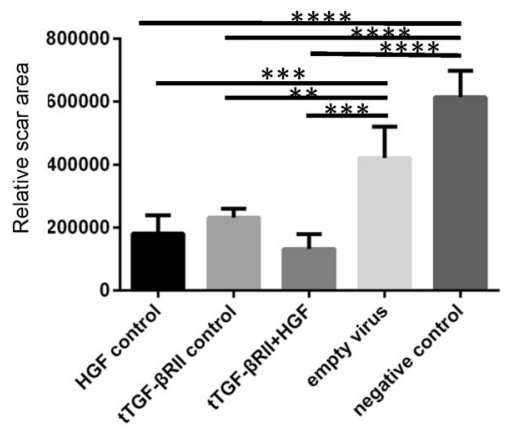

B

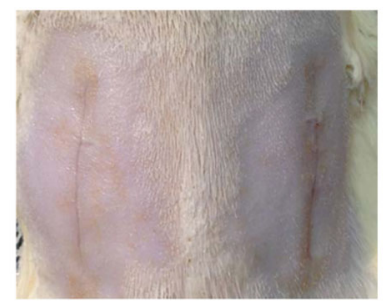

Figure 4. Relative scar area in each group. A, Quantification of the relative wound scar area revealed that the wounds in the experimental (t-TGF- $\beta$ RII + HGF), HGF control, and t-TGF$\beta R$ II groups healed with less scarring compared with those in the empty virus and blank groups. The result was most prominent in the experimental group. Data are reported as means \pm SD. $\quad{ }^{* *} P<0.01, \quad{ }^{* * *} P<0.001, \quad{ }^{* * * *} P<0.0001$ (Student-Newman-Keuls test). B, On macroscopic inspection, the treated wound (left side) exhibited less visible scarring compared with the control wound (right side) at 14 days after wounding. tTGF- $\beta$ RII: truncated transforming growth factor$\beta$ type II receptor; HGF: hepatocyte growth factor.

\section{Discussion}

Wound healing is a complicated process that has only started to become elucidated (8). Scar formation has been conventionally considered a natural event during the process of wound healing. A number of studies have reported that TGF- $\beta$ signaling plays a fundamental role in pathological fibroproliferation and abnormal scar formation $(1,9,10)$. During the process of wound healing, TGF- $\beta$ binds to and activates specific receptors located on the cell membrane. The ligand TGF- $\beta$ assembles a receptor complex that activates the Smad proteins, and these proteins assemble multi subunit complexes that regulate transcription (3).

Strategies for scar reduction have been adopted based on targeting of the TGF- $\beta$ signaling pathway. Shah et al. (11) demonstrated that wounds in adult rodents could be modified to reduce scar formation using a TGF- $\beta$ neutralizing antibody, providing direct evidence that manipulating wound TGF- $\beta$ can inhibit scarring. In addition, wounds in TGF- $\beta$ knockout mice heal with less formation of granulation tissue and faster epithelialization compared with control mice (12). Numerous studies have demonstrated that blocking the TGF- $\beta$ signaling pathway may be more effective in inhibiting TGF- $\beta$-mediated scar formation $(2-4,13,14)$.

Systemic pharmacological inhibitors of collagen synthesis have been used clinically, with significant systemic adverse effects (15). Therefore, it is necessary to develop a method for inhibiting scar formation without inhibition of wound healing. HGF is a multifunctional growth factor, which can promote mitosis, has anti-apoptotic, anti-fibrotic, and pro-angiogenic properties, inhibits fibrosis and generation of TGF- $\beta$ in hepatic tissue, and enhances collagenase activity (6). It has been suggested that HGF prevents fibrosis in liver and lung injury models (16-18), whereas other studies revealed that HGF prevents fibrosis after injury or surgery and facilitates tissue regeneration in wounds $(6,19,20)$.

The advantages of gene therapy are the higher efficiency, fewer side effects, and a long expression time in wounds. Ozawa et al. (21) used combination gene therapy with HGF and t-TGF- $\beta$ RII for rat liver cirrhosis following partial hepatectomy and suggested that gene therapy may increase the possibility of hepatectomy in a cirrhotic liver by improving fibrosis, hepatic function, and hepatocyte regeneration. In the present study, a similar strategy was applied for gene therapy of wound scarring. Incisional wounding was performed in rats and double gene (t-TGF- $\beta$ RII//HGF) complexes were successfully transfected into NIH-3T3 mouse embryonic fibroblasts by lentivirus to study the effectiveness of blocking TGF- $\beta$ signaling combined with HGF.

In our in vitro experiments, the expression of collagen I and III in the experimental group was found to be significantly reduced compared with that in the blank and negative control groups. The proliferation of NIH-3T3 cells, which was quantified by the CCK-8 assay, was significantly inhibited by the transfection of t-TGF- $\beta$ RIII/ HGF-lentivirus. In vivo gene therapy in the rat model demonstrated that the expression of collagen I and III in the experimental group was significantly reduced compared with that in the control groups.

Based on the above-mentioned data, it may be concluded that fibrosis and wound scarring were inhibited by the cotransfection of HGF and t-TGF- $\beta$ RII. Furthermore, histological examination and qualification revealed that the width of the scar was markedly narrower in the experimental group compared with that in the control groups, including the single t-TGF- $\beta$ RII control group. These findings support that the combination gene therapy of t-TGF- $\beta$ RII and HGF promoted wound healing with less scarring and more epithelial tissue formation, not only by suppressing the overgrowth of collagen due to its antifibrotic effect, but also by promoting tissue regeneration. However, HGF expression needs to be further optimized and, in addition, since we used lentiviral vector to deliver two target genes, the effects of continued expression of these two molecules need to be further investigated. A vector with on/off switch features can be also tested in this animal model. 


\section{Acknowledgments}

The present study was funded by the National Natural Science Foundation of China (No. 81671918), Zhejiang

\section{References}

1. Wang R, Ghahary A, Shen Q, Scott PG, Roy K, Tredget EE. Hypertrophic scar tissues and fibroblasts produce more transforming growth factor-beta1 mRNA and protein than normal skin and cells. Wound Repair Regen 2000; 8: 128137, doi: 10.1046/j.1524-475x.2000.00128.x.

2. Lindblad WJ. Gene therapy in wound healing--2000: a promising future. Wound Repair Regen 2000; 8: 441-442, doi: 10.1046/j.1524-475x.2000.00249.x.

3. Massagué J, Wotton D. Transcriptional control by the TGFbeta/Smad signaling system. EMBO J 2000; 19(8): 17451754, doi: 10.1093/emboj/19.8.1745.

4. Yamamoto $\mathrm{H}$, Ueno $\mathrm{H}$, Ooshima A, Takeshita A. Adenovirus-mediated transfer of a truncated transforming growth factor-beta (TGF-beta) type II receptor completely and specifically abolishes diverse signaling by TGF-beta in vascular wall cells in primary culture. J Biol Chem 1996; 271: 16253 16259, doi: 10.1074/jbc.271.27.16253.

5. Liu W, Chua C, Wu X, Wang D, Ying D, Cui L, et al. Inhibiting scar formation in rat wounds by adenovirus-mediated overexpression of truncated TGF-beta receptor II. Plast Reconstr Surg 2005; 115: 860-870, doi: 10.1097/01.PRS. 0000153037.12900.45.

6. Ono I, Yamashita T, Hida T, Jin HY, Ito Y, Hamada H, et al. Local administration of hepatocyte growth factor gene enhances the regeneration of dermis in acute incisional wounds. J Surg Res 2004; 120: 47-55, doi: 10.1016/j.jss. 2003.08.242.

7. Wu X, Gao Z, Song N, Chua C, Deng D, Cao Y, et al. Creating thick linear scar by inserting a gelatin sponge into rat excisional wounds. Wound Repair Regen 2007; 15: 595606, doi: 10.1111/j.1524-475X.2007.00256.x.

8. Rhett JM, Ghatnekar GS, Palatinus JA, O'Quinn M, Yost MJ, Gourdie RG. Novel therapies for scar reduction and regenerative healing of skin wounds. Trends Biotechnol 2008; 26: 173-180, doi: 10.1016/j.tibtech.2007.12.007.

9. Bettinger DA, Yager DR, Diegelmann RF, Cohen IK. The effect of TGF-beta on keloid fibroblast proliferation and collagen synthesis. Plast Reconstr Surg 1996; 98: 827-833, doi: 10.1097/00006534-199610000-00012.

10. Singer AJ, Clark RA. Cutaneous wound healing. $N$ Engl $J$ Med 1999; 341: 738-746, doi: 10.1056/NEJM1999090234 11006.

11. Shah M, Foreman DM, Ferguson MW. Neutralising antibody to TGF-beta 1,2 reduces cutaneous scarring in adult rodents. J Cell Sci 1994; 107: 1137-1157.

12. Koch RM, Roche NS, Parks WT, Ashcroft GS, Letterio JJ, Roberts $A B$. Incisional wound healing in transforming growth
Provincial Medical and Healthy Science Foundation of China (No. 2019ZD028 and No. 2018KY874), and National Key Research Program of China (No. 2016YFC1101004).

factor-beta1 null mice. Wound Repair Regen 2000; 8: 179191, doi: 10.1046/j.1524-475x.2000.00179.x.

13. Ashcroft GS, Yang $X$, Glick AB, Weinstein M, Letterio JL, Mizel DE, et al. Mice lacking Smad3 show accelerated wound healing and an impaired local inflammatory response. Nat Cell Biol 1999; 1: 260-266, doi: 10.1038/ 12971.

14. Tan WQ, Gao ZJ, Xu JH, Yao HP. Inhibiting scar formation in vitro and in vivo by adenovirus-mediated mutant Smad4: a preliminary report. Exp Dermatol 2011; 20: 119-124, doi: 10.1111/j.1600-0625.2010.01186.x.

15. Henderson J, Ferguson MW, Terenghi G. The reinnervation pattern of wounds and scars after treatment with transforming growth factor beta isoforms. J Plast Reconstr Aesthet Surg 2012; 65: e80-e86, doi: 10.1016/j.bjps.2011. 12.013

16. Matsuda Y, Matsumoto K, Ichida T, Nakamura T. Hepatocyte growth factor suppresses the onset of liver cirrhosis and abrogates lethal hepatic dysfunction in rats. $J$ Biochem 1995; 118: 643-649, doi: 10.1093/oxfordjournals.jbchem. a124958.

17. Yaekashiwa M, Nakayama S, Ohnuma K, Sakai T, Abe T, Satoh K, et al. Simultaneous or delayed administration of hepatocyte growth factor equally represses the fibrotic changes in murine lung injury induced by bleomycin. A morphologic study. Am J Respir Crit Care Med 1997; 156: 1937-1944, doi: 10.1164/ajrccm.156.6.9611057.

18. Matsuda $Y$, Matsumoto $K$, Yamada A, Ichida T, Asakura $H$, Komoriya $Y$, et al. Preventive and therapeutic effects in rats of hepatocyte growth factor infusion on liver fibrosis/ cirrhosis. Hepatology 1997; 26: 81-89, doi: 10.1002/hep. 510260111.

19. Akasaka $\mathrm{Y}$, Fujita K, Ishikawa $\mathrm{Y}$, Asuwa N, Inuzuka K, Ishihara $\mathrm{M}$, et al. Detection of apoptosis in keloids and a comparative study on apoptosis between keloids, hypertrophic scars, normal healed flat scars, and dermatofibroma. Wound Repair Regen 2001; 9: 501-506, doi: 10.1046/ j.1524-475x.2001.00501.x.

20. Xiao Z, Xi C. Hepatocyte growth factor reduces hypertrophy of skin scar: in vivo study. Adv Skin Wound Care 2013; 26: 266-270, doi: 10.1097/01.ASW.0000429705. 02588.f5.

21. Ozawa S, Uchiyama K, Nakamori M, Ueda K, Iwahashi M, Ueno $\mathrm{H}$, et al. Combination gene therapy of HGF and truncated type II TGF-beta receptor for rat liver cirrhosis after partial hepatectomy. Surgery 2006; 139: 563-573, doi: 10.1016/j.surg.2005.10.003. 